\section{Evaluation of the effect of JPEG and JPEG2000 image compression on the detection of diabetic retinopathy}

\author{
Abstract \\ Aims To compare the effect of classic Joint \\ Photographic Experts Group (JPEG) and \\ JPEG2000 compression algorithms on \\ detection of diabetic retinopathy (DR) lesions. \\ Methods In total, 45 colour fundus \\ photographs obtained with a digital \\ nonmydriatic fundus camera were saved in \\ uncompressed Tagged Interchanged Files \\ Format (TIFF) format (1.26 MB). They were \\ graded jointly by two retinal specialists at a \\ 1 month interval for soft exudates, hard \\ exudates, macular oedema, newvessels, \\ intraretinal microvascular abnormalities \\ (IRMA), and retinal haemorrhages and/or \\ microaneurysms. They were compressed to \\ $118,58,41$, and $27 \mathrm{~KB}$ by both algorithms and \\ $24 \mathrm{~KB}$ by classic JPEG, placed in random order \\ and graded again jointly by the two retina \\ specialists. Subjective image quality was \\ graded, and sensitivity, specificity, positive \\ and negative predictive values, and kappa \\ statistic were calculated for all lesions at all \\ compression ratios. \\ Results Compression to $118 \mathrm{~KB}$ showed no \\ effect on image quality and kappa values were \\ high (0.94-1). Image degradation became \\ important at $27 \mathrm{~KB}$ for both algorithms. At \\ high compression levels, IRMA and HMA \\ detection were most affected with JPEG2000 \\ performing slightly better than classic JPEG. \\ Conclusion Performance of classic JPEG and \\ JPEG2000 algorithms is equivalent when \\ compressing digital images of DR lesions from \\ 1.26 MB to $118 \mathrm{~KB}$ and $58 \mathrm{~KB}$. Higher \\ compression ratios show slightly better results \\ with JPEG2000 compression, but may be \\ insufficient for screening purposes. \\ Eye (2007) 21, 487-493. doi:10.1038/sj.eye.6702238; \\ published online 3 February 2006
}

J Conrath ${ }^{1,2}$, A Erginay', R Giorgi ${ }^{3}$,

A Lecleire-Collet ${ }^{1}$, E Vicaut ${ }^{4}$, J-C Klein ${ }^{5}$,

A Gaudric ${ }^{1}$ and P Massin ${ }^{1}$

Keywords: diabetic retinopathy; digital fundus photography; JPEG; JPEG2000

Telescreening for diabetic retinopathy (DR) is relevant to compensate for the lack of ophthalmologists assessing DR, especially in remote areas. Digital fundus photography is commonly used for DR screening. ${ }^{1}$ High-quality digital images can reach a size of $1.5 \mathrm{MB}$ or greater. Compression techniques are required to fasten transmission of such images, yet information may be lost during compression. Standards have been defined for radiology and pathology; yet to date, resolution levels required for reliable diagnosis in ophthalmology have not been determined.

Basu et $a l^{2}$ evaluated the effect of classic Joint Photographic Experts Group (JPEG)

compression (cJPEG) on the grading of DR lesions and found that compression ratios of $1: 20$ to $1: 12$ were acceptable. JPEG2000, a new image compression algorithm purportedly rendering higher quality images than that of cJPEG at higher compression ratios, has been evaluated for the compression of medical images in radiology., ${ }^{3,4}$ The aim of our study was to compare cJPEG and JPEG2000 compression on the detection of DR lesions.

\section{Methods}

In total, 45 good quality digital images including different pathologic lesions of DR were selected from our digital image bank. Good quality was defined as a well-centred image without dark or bright peripheral halos, in focus that allowed unambiguous analysis of fine details. They were obtained with Topcon's nonmydriatic retinal fundus camera (45 opening, TRC-NW6, Topcon Europe,
${ }^{1}$ Department of

Ophthalmology, Hôpital Lariboisière, Assistance Publique - Hôpitaux de Paris, Université Paris 7, Paris, France

${ }^{2}$ Department of Ophthalmology, Hôpital de la Timone, Assistance Publique - Hôpitaux de Marseille, Université de la Méditerranée, Marseille, France

${ }^{3}$ LERTIM, Faculté de Médecine de Marseille Marseille, France

${ }^{4}$ Unité de Recherche Clinique, Hôpital Lariboisière, Assistance Publique - Hôpitaux de Paris, Université Paris 7 , Paris, France

${ }^{5}$ Centre de Morphologie Mathématique, Ecole des Mines, Fontainebleau,

France

Correspondence: P Massin, Department of

Ophthalmology, Hôpital Lariboisière, 2 rue Ambroise Paré, F-75010 Paris, France Tel: + 331499 52475; Fax: + 33149956484 .

E-mail: p.massin@

Irb.aphp.fr

Received: 27 April 2005 Accepted in revised form: 29 November 2005 Published online: 3 February 2006

None of the authors have any commercial relationship with any of the companies mentioned 
Rotterdam, The Netherlands) connected to a tri-CCD colour video camera (Sony, DXC-950 P, Tokyo, Japan). Images were captured without pupil dilation, in true colour ( 24 bits) at a resolution of $800 \times 600$ pixels resulting in an uncompressed image size of $1.26 \mathrm{MB}$.

Photographs were selected to ensure adequate distribution of different DR lesions; eight photographs showed normal fundus, 37 various numbers of haemorrhages/microaneurysms (HMA), 18 soft exudates (SE), 22 hard exudates (HE), seven new vessels on the disk (NVD) or elsewhere (NVE), and four showed intraretinal microvascular abnormalities (IRMA). Images were stored as uncompressed TIFF (tagged interchanged files format) files. This study of retrospective design adhered to the tenets of the declaration of Helsinki.

\section{Compression}

The 45 TIFF images were first compressed to five different levels, using the CJPEG algorithm with PhotoShop 5.0 (Adobe, San Jose, CA, USA), to 118, 58, 41, 27 , and $24 \mathrm{~KB}$ in size (representing compression ratios of $1: 11,1: 22,1: 31,1: 47$, and $1: 53$, respectively). The TIFF images were then compressed by the JPEG2000 algorithm using the ImagePress JP2 plug-in (Pegasus, Tampa, FL, USA) for Photoshop computer software at levels $1: 11,1: 22,1: 31$, and $1: 47$, resulting in image sizes of $118,58,41$ and $27 \mathrm{~KB}$, respectively. These weights were chosen as it became empirically clear that the low compression ratio $(118 \mathrm{~KB})$ and high compression ratio (27 KB) were respectively of excellent and poor quality with both compression algorithms. For classic JPEG images, only $24 \mathrm{~KB}$ was applied as it appeared much worse than the others, and the gain in storage/ transmission size between 27 and $24 \mathrm{~KB}$ is negligible. All 405 compressed images were placed in a random order.

\section{Image grading}

Images were displayed on a 21-inch monitor (resolution: $1280 \times 1024 \times 24$ bits, CRT Sony Trinitron Multiscan G500, Tokyo, Japan). To avoid intergrader variability, all images were graded jointly by two retinal specialists. No image processing was used. Each sign of DR was graded on TIFF photographs as SE, HE, 'macular oedema' (ME, which used as a surrogate the presence of HE within one disc diameter of the foveola), NVD, NVE, and IRMA were assessed as absent (0), questionable (1) or present (2). HMA were graded as (0), absent; (1), questionable or less than 5 ; (2), 5 to 10, and (3), more than 10. TIFF images were graded twice at an interval of 1 month in order to assess intragrader variability. Just after the second TIFF grading, compressed images $(n=405)$ were then presented in random order and graded jointly by the two graders. Image quality was graded as being good (image degradation not apparent), acceptable (image degradation apparent, but still allowing subjectively reliable assessment), or poor (quality not sufficient from which to make reliable assessment). Then each lesion of DR was graded on each image according to the above scale.

\section{Statistics}

TIFF photographs were the reference standard. However, some variability was observed between the two successive gradings due to intragrader variability. A consensus grade for each image was reached after discussion between the two graders, to establish the gold standard.

Lesion grades for each image at each compression level were then compared with the gold standard, and sensitivity, specificity, positive and negative predictive values were calculated. For sensitivity and specificity, the score for each lesion was dichotomized as present or absent. Questionable lesions were conventionally considered positive. 95\% confidence intervals were calculated. A weighted kappa statistic of each compression grade was calculated to evaluate level of agreement between gold standard and grading scores for each level of compression. Kappa statistic agreement was termed slight $(0-0.20)$, fair $(0.21-0.40)$, moderate (0.41$0.60)$, substantial (0.61-0.80), and almost perfect ( 0.81 and more). ${ }^{5}$ Agreement between gold standard and grading scores for HMA was also calculated, when HMA were absent (grade 0) vs present (grades 1, 2, and 3), to study the effect of compression on distinction of no lesions to few or many. Kappa statistics were also used to assess intragrader agreement between the two gradings at a 1-month interval. The SPSS v11.1 software statistical package (SPSS, Chicago, IL, USA) for Windows was used.

\section{Results}

Table 1 summarizes image quality assessment for different compression levels. With cJPEG image quality started to decrease at $41 \mathrm{~KB}$ and blocking effects were obvious in almost all $24 \mathrm{~KB}$ images. With JPEG2000, at sizes of 27 and $41 \mathrm{~KB}$, slightly more 'poor' images were seen than that for cJPEG, with 'rice grain' artefacts present.

No intragrader variability was observed when grading for SE, HE, ME, NVD/NVE, and IRMAs on TIFF images. Kappa statistic for intragrader agreement for the grading of HMA on TIFF images was 0.87 (95\% confidence interval, 0.74-0.99). 
Table 1 Values of subjective image quality assessment for 45 compressed images at different levels of cJPEG and JPEG 2000 compression

\begin{tabular}{|c|c|c|c|c|c|c|c|c|c|}
\hline \multirow[b]{2}{*}{ Image size } & \multicolumn{5}{|c|}{ Classic JPEG } & \multicolumn{4}{|c|}{ JPEG2000 } \\
\hline & $24 K B$ & $27 \mathrm{~KB}$ & $41 \mathrm{~KB}$ & $58 \mathrm{~KB}$ & $118 \mathrm{~KB}$ & $27 K B$ & $41 \mathrm{~KB}$ & $58 \mathrm{~KB}$ & $118 \mathrm{~KB}$ \\
\hline$Q$ value & 10 & 20 & 40 & 60 & 80 & & & & \\
\hline Compression ratio & $1: 53$ & $1: 47$ & $1: 31$ & $1: 22$ & $1: 11$ & $1: 47$ & $1: 31$ & $1: 22$ & $1: 11$ \\
\hline Quality good & 0 & 0 & 29 & 45 & 45 & 0 & 22 & 41 & 45 \\
\hline Acceptable & 5 & 38 & 15 & 0 & 0 & 33 & 15 & 4 & 0 \\
\hline Poor & 40 & 7 & 1 & 0 & 0 & 12 & 8 & 0 & 0 \\
\hline
\end{tabular}

Table 2 Sensitivity, specificity, positive predictive value, and negative predictive value for 45 compressed images at different levels of cJPEG and JPEG 2000 compression

\begin{tabular}{|c|c|c|c|c|c|c|c|c|c|}
\hline \multirow[b]{2}{*}{ Image size } & \multicolumn{6}{|c|}{ Classic JPEG } & \multicolumn{3}{|c|}{ JPEG2000 } \\
\hline & $24 K B$ & $27 \mathrm{~KB}$ & $41 \mathrm{~KB}$ & $58 \mathrm{~KB}$ & $118 \mathrm{~KB}$ & $27 \mathrm{~KB}$ & $41 \mathrm{~KB}$ & $58 \mathrm{~KB}$ & $118 \mathrm{~KB}$ \\
\hline \multirow[t]{2}{*}{$Q$ value } & 10 & 20 & 40 & 60 & 80 & jp2 & jp2 & jp2 & Jp2 \\
\hline & Sens/Spe & Sens/Spe & Sens/Spe & Sens/Spe & Sens/Spe & Sens/Spe & Sens/Spe & Sens/Spe & Sens/Spe \\
\hline HMA & $92 / 75$ & $89 / 75$ & $95 / 100$ & $95 / 100$ & $100 / 100$ & $97 / 50$ & $83 / 88$ & $95 / 100$ & $100 / 100$ \\
\hline Soft exudates & $89 / 96$ & $83 / 100$ & $100 / 100$ & $100 / 100$ & $100 / 100$ & $89 / 100$ & $89 / 100$ & $94 / 96$ & $100 / 100$ \\
\hline Hard exudates & $95.5 / 100$ & $95.5 / 100$ & $95.5 / 100$ & $100 / 100$ & $100 / 100$ & $95.5 / 100$ & $95.5 / 100$ & $95.5 / 100$ & $100 / 100$ \\
\hline $\mathrm{ME}$ & $100 / 100$ & $100 / 100$ & $100 / 100$ & $100 / 100$ & $100 / 100$ & $100 / 97$ & $100 / 100$ & $100 / 97$ & $100 / 100$ \\
\hline IRMA & $25 / 92.5$ & $50 / 100$ & $50 / 100$ & $100 / 100$ & $100 / 100$ & $75 / 100$ & 75/100 & $75 / 100$ & $100 / 100$ \\
\hline NVE & $83 / 100$ & $100 / 100$ & $100 / 100$ & $100 / 100$ & $100 / 100$ & $83 / 97$ & $100 / 97$ & $100 / 97$ & $100 / 100$ \\
\hline \multirow[t]{2}{*}{ NVD } & $100 / 100$ & $100 / 100$ & $100 / 100$ & $100 / 100$ & $100 / 100$ & $100 / 100$ & $100 / 100$ & $100 / 100$ & $100 / 100$ \\
\hline & PPV/NPV & $\mathrm{PPV} / \mathrm{NPV}$ & $\mathrm{PPV} / \mathrm{NPV}$ & $\mathrm{PPV} / \mathrm{NPV}$ & $\mathrm{PPV} / \mathrm{NPV}$ & $\mathrm{PPV} / \mathrm{NPV}$ & PPV/NPV & $\mathrm{PPV} / \mathrm{NPV}$ & $\mathrm{PPV} / \mathrm{NPV}$ \\
\hline HMA & $94.5 / 66.5$ & $94 / 60$ & $100 / 80$ & $100 / 80$ & $100 / 100$ & $90 / 80$ & $97 / 70$ & $100 / 80$ & $100 / 100$ \\
\hline Soft exudates & 94/93 & $100 / 90$ & $100 / 100$ & $100 / 100$ & $100 / 100$ & $100 / 93$ & $100 / 93$ & $94 / 96$ & $100 / 100$ \\
\hline Hard exudates & $100 / 96$ & $100 / 96$ & $100 / 96$ & $100 / 100$ & $100 / 100$ & $100 / 96$ & $100 / 96$ & $100 / 96$ & $100 / 100$ \\
\hline $\mathrm{ME}$ & $100 / 100$ & $100 / 100$ & $100 / 100$ & $100 / 100$ & $100 / 100$ & $91 / 100$ & $100 / 100$ & $91 / 100$ & $100 / 100$ \\
\hline IRMA & $25 / 93$ & $100 / 95$ & $100 / 95$ & $100 / 100$ & $100 / 100$ & $100 / 98$ & $100 / 98$ & $100 / 98$ & $100 / 100$ \\
\hline NVE & $100 / 97.5$ & $100 / 100$ & $100 / 100$ & $100 / 100$ & $100 / 100$ & $83 / 97$ & $86 / 100$ & $86 / 100$ & $100 / 100$ \\
\hline NVD & $100 / 100$ & $100 / 100$ & $100 / 100$ & $100 / 100$ & $100 / 100$ & $100 / 100$ & $100 / 100$ & $100 / 100$ & $100 / 100$ \\
\hline
\end{tabular}

Sensitivity, specificity, positive and negative predictive values are given in Table 2 . Table 3 summarizes agreement of lesion grade between TIFF and compressed images.

Compression had no effect on the detection of NVD. Among NVE, two were subtle and smaller than $1 / 2$ disc area in size; one of them was not detected on both $24 \mathrm{~KB}$ cJEPG and $27 \mathrm{~KB}$ JPEG2000 images. 27, 41 and 58 KB JPEG2000 image sizes led to identification of one false-positive NVE.

Good-to-excellent agreement was observed for detection of exudates between TIFF and compressed images. Only subtle isolated exudates were missed in 24-41 KB cJPEG and 27-58 KB JPEG2000 images. For SE at 41 and $58 \mathrm{~KB}$, JPEG2000 showed lower sensitivity.

The lowest level of agreement was associated with IRMA, present on four images; there were many of them on two, and few small, isolated ones on the two others. In cJPEG, small IRMA were missed at 27 and $41 \mathrm{~KB}$. At these same image sizes, JPEG2000 performed slightly better for IRMA.

Kappa statistics analysis for HMA grades showed fairto-good agreement (greater than 0.5) between TIFF and either JPEG images, at any compression level. However, variability of HMA grades was both due to intragrader variability and compression. For $118 \mathrm{~KB}$ size in both cJPEG and JPEG2000, variability was mostly due to intragrader variability (kappa values being greater than that for intragrader agreement). For other cases, variability was mostly due to compression, as kappa values were smaller than for intragrader agreement.

Kappa values were higher when considering absence vs presence of HMA than that of global detection for all images, except for the 27 and 41 KB JPEG2000 images. 


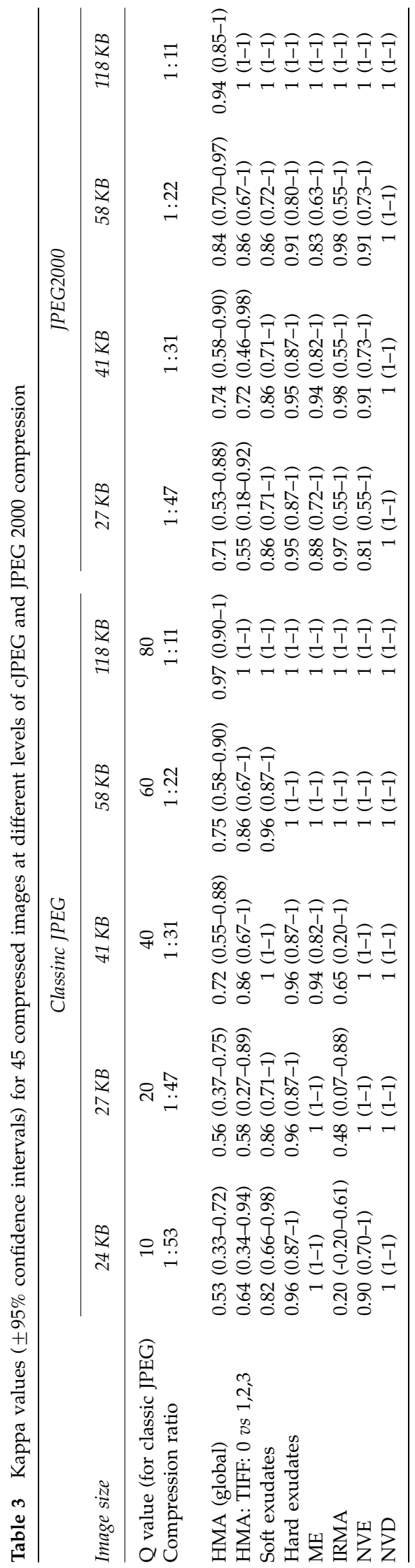

\section{Discussion}

Compression reduces image file size, allowing quicker transmission and using less storage space. The Joint Photographic Experts Group (JPEG) compression is the most common image format; it is largely used for medical imaging. It is a 'lossy' compression technique, meaning that some information and image quality are lost during compression. The amount of information that is discarded determines the amount of compression. The cJPEG algorithm breaks the image into $8 \times 8$ pixel blocks and performs a discrete cosine transform on each block. An $8 \times 8$ block of spectral coefficients with most of the information concentrated in relatively few coefficients is obtained. Quantization is then performed, closely preserving low-frequency components, approximating high-frequency components. The amount of discarded information determines compression level. A coding process compresses the remaining frequency coefficients.

The JPEG2000 wavelet algorithm uses a different approach, dividing the image into a series of nonoverlapping rectangular blocks called tiles. ${ }^{6}$ Each tile component is decomposed using the wavelet transforms into decomposition levels, each of which contains a number of subbands. These subbands contain information describing the horizontal and vertical characteristics of the original tile. They are computed using a one-dimensional filter applied in both directions. This gives four smaller image blocks; one with low resolution, one with high vertical and low horizontal resolution, one with low vertical and high horizontal resolution, one with all high resolution. This application of one-dimensional filters in both directions is then repeated a number of times on the low-resolution image block (dyadic decomposition). After transformation, all coefficients are quantized. This is the process by which the coefficients are reduced in precision. Following quantization, each subband is subjected to a packet partition or code-blocks creation, ${ }^{7}$ which are the fundamental entities used for the final step of entropy coding.

The effect of compression on retinal images has been investigated in a number of studies. Eikelboom $e t a l^{8}$ studied the effect of various levels of both cJPEG and wavelet compression on the quality of digitized retinal images and using different methods concluded that a digital image 1.5 MB in size could be compressed to $29 \mathrm{~KB}$ without serious degradation in quality. Newsom et $a l^{9}$ demonstrated significant loss of sensitivity to the features of DR with cJPEG compression of $35 \mathrm{~mm}$ slides that had been digitized concluding that this was due to the TFT screen they used. Only retinopathy level was considered, with no mention of individual lesion counts. 

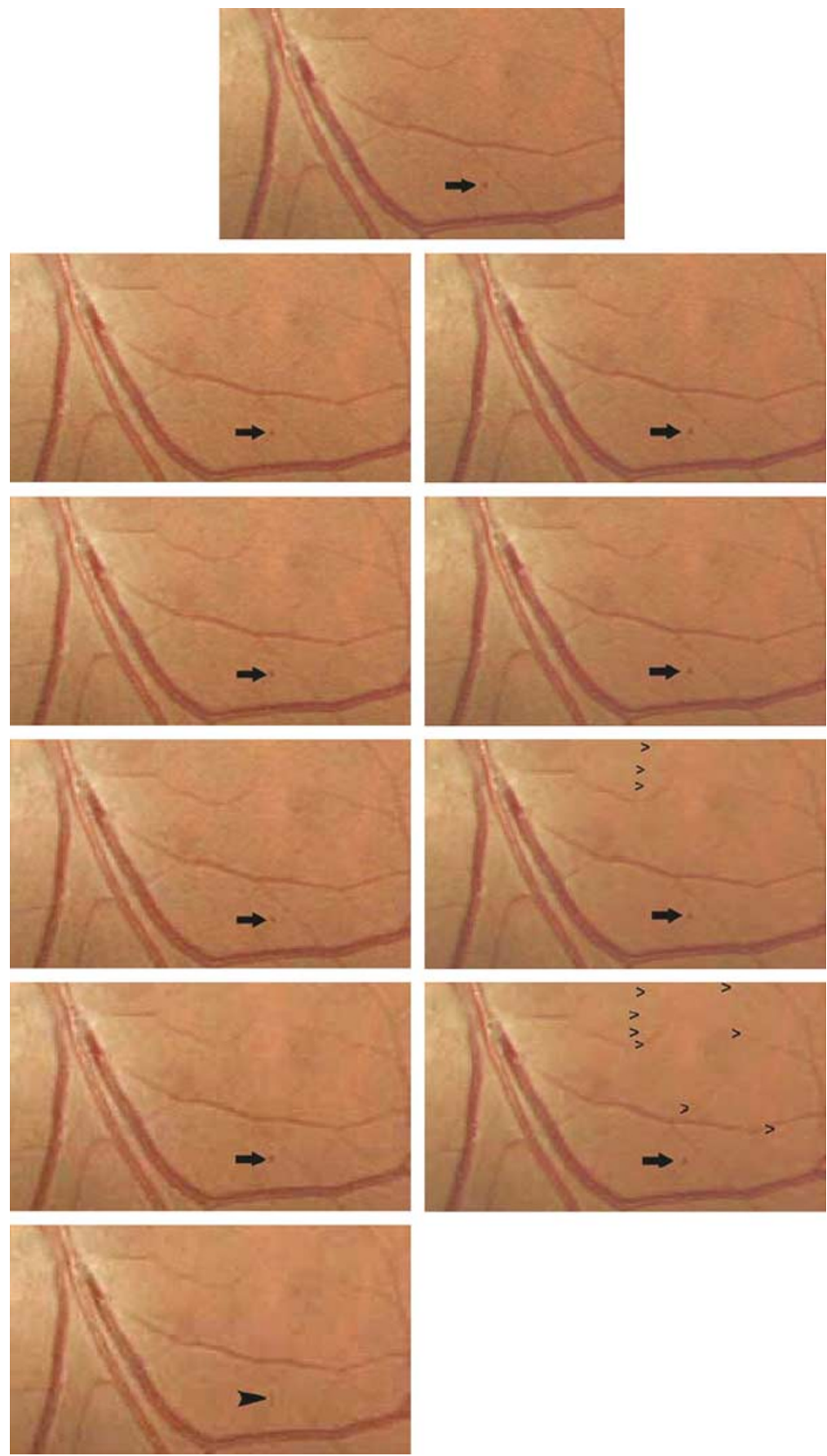

Figure 1 Examples of the effect on a retinal image (detail) of the different JPEG and JEPG2000 compression ratios. Top Middle: Original 1.26 MB TIFF image. Left column, from top to bottom: classic JPEG compression to 118, 58, 41, 27 and $24 \mathrm{~KB}$. Right column, from top to bottom: JPEG2000 wavelet compression to 118, 58, 41 and $27 \mathrm{~KB}$. A small red dot (HMA, arrow) is seen in all images except the $24 \mathrm{~KB}$ JPEG image (bottom left), where it becomes a vertical line, part of a blocking artefact (large arrowhead). The vessels surrounding it, seen well on TIFF and both $118 \mathrm{~KB}$ images progressively fade away with both compression algorithms. Rice-grain artefacts become more prominent in the JPEG2000 images as image size decreases (small arrowheads in 41 and $27 \mathrm{~KB}$ images).

Basu $e t a l^{2}$ explored the effect of four different levels of cJPEG compression on 58 digitally acquired fundus images, finding that up to $1: 20$ compression ratios were acceptable. Stellingwerf $e t a l^{10}$ compared uncompressed
TIFF and compressed cJPEG digitally acquired fundus photographs with $35 \mathrm{~mm}$ retinal slides, finding $1: 30$ compression decreased sensitivity from $0.86-0.92$ to $0.72-0.74$. Using large $2008 \times 3040$ pixel images, Baker 
found $1: 55$ and $1: 113$ compression ratios acceptable for DR screening. ${ }^{11}$

The aim of our study was to compare cJPEG and JPEG2000 compression algorithms in screening for DR lesions. We therefore chose individual images to determine effect of compression on specific lesions and not composite fundus images as ETDRS grading was not our aim.

In cJPEG compression, blocking artefacts started to be visible on $41 \mathrm{~KB}$-images (Figure 1) and became obvious on $27 \mathrm{~KB}$ images. Image degradation was first noticed at an earlier level of compression than that in Eikelboom's study. ${ }^{8}$ In JPEG2000 images, certain images were subjectively found to be blurred at all three levels of compression, without typical 'blocking' artefacts, yet artefacts specific to JPEG2000 ('wavelet or rice-shaped', ${ }^{12}$ Figure 1) were observed. Another feature visible on JPEG2000 images at high compression ratios was the 'smoothing' effect; as Eikelboom et al ${ }^{8}$ noted wavelet images may be pleasant to look at, even if appearing somewhat fuzzy or 'out of focus'. ${ }^{8}$

With the levels of compression that we used, we observed relatively few effects on the detection of gross anomalies, which were detected with good sensitivity. Eikelboom et $\mathrm{al}^{8}$ found that large anomalies could be detected on retinal images at a compression ratio over $1: 300$, using JPEG. In our study, NVD or NVE were detected at any level of JPEG compression when they were greater than $1 / 2$ disc area in size. However, smaller new vessels were missed in one case each on $24 \mathrm{~KB}$ cJPEG and $27 \mathrm{~KB}$ JPEG2000 images.

$\mathrm{HE}$ were well detected at any level of either compression. Indeed, HE are small lesions, but are often grouped together in clusters or large circinate rings. Only small isolated HE were missed at higher levels of compression.

As expected, the effect of compression was more pronounced on small, subtle, low-contrasted anomalies. Vanishing SE were missed at the lowest compression levels. The lowest level of agreement was associated with IRMA; small, isolated IRMA present on two images were missed from $41 \mathrm{~KB}$ images. This was the case in one JPEG2000 image.

An important point in this study was the effect of image compression on the detection of microaneurysms; they are the first ophthalmoscopic sign of early DR, and their detection is particularly critical when screening for DR; it is important to be able to count them at early stages of DR to follow the progression of the disease. ${ }^{13}$ The possibility to detect and count them properly on compressed images will thus determine the highest compression level clinically acceptable to screen for DR.

The sensitivity to detect microaneurysms is not as good on digital photography as on conventional 35-mm photography, although several authors have found good agreement between both techniques for DR grading, ${ }^{14-16}$ the poorest agreement between DR grades was recorded at level 21, characterized by the presence of few microaneurysms. ${ }^{17-19}$ This is due to the lower resolution of digital photography, which makes the images granular and increases the number of questionable lesions when grading for microaneurysms. Image compression is expected to increase this phenomenon, as well as the difficulty in detecting and counting microaneurysms. For both classic and JPEG2000, global level of agreement between $118 \mathrm{~KB}$ compressed and TIFF images grading was almost perfect. Image compression did not lead to increased variability, compared to variability observed when grading TIFF images twice. At intermediate compression ratios (41 and $58 \mathrm{~KB}$ ), global performance assessed by the kappa statistic was quite similar for both algorithms. At $27 \mathrm{~KB}$, JPEG2000 performed slightly better than CJPEG for distinction of different HMA levels. At these levels of compression, cJPEG compression resulted in a significant decrease of visibility of critical details due to blocking artefacts that affected microaneurysm grading. Thus, such levels of compression with cJPEG do not appear suitable for DR screening.

Blocking artefacts are an intrinsic limitation of the cJPEG algorithm that splits the image in blocks of $8 \times 8$ pixels. Low-quality compressed images are inefficiently compressed because for each block, only the lowest frequency (constant) component remains, and these various components are encoded inefficiently. As the JPEG2000 standard works on the image as a whole, it does not present blocking effects, as it is based on wavelet decompositions. However, implementation issues have also imposed that this newer JPEG standard splits the image into 'tiles', 6 , so that the problem of artefacts ('wavelet or rice-shaped') still remain. On the other hand, at high compression ratios, JPEG2000 might be more efficient in retaining small details like microaneurysms than the current cJPEG standard, especially when these details are much smaller than the $8 \times 8$ block size. This effect is supported by our finding a higher kappa for $27 \mathrm{~KB}$ JPEG2000 vs cJPEG images for global HMA evaluation.

In spite of higher performance computers, more sophisticated software and high-speed internet-based data transmission, compression will still be a matter of concern for telemedicine in the future, as digital retinal screening cameras are also getting higher definitions (above the $5 \mathrm{MegaPixel}$ range to date). We found that cJPEG as well as JPEG2000 compression of a $1.26 \mathrm{MB}$ fundus image to $118 \mathrm{~KB}$ does not affect accuracy when compared to uncompressed TIFF images. At higher compression ratios, JPEG2000 has proven superior to cJPEG in radiology; ${ }^{4}$ we also found it slightly better, but 
also with results insufficient for clinical use. ${ }^{4}$ This remains to be tested in a clinical setting using larger image sizes.

\section{References}

1 Freudenstein U, Verne J. A national screening programme for diabetic retinopathy. BMJ 2001; 323: 4-5.

2 Basu A, Kamal AD, Illahi W, Khan M, Stavrou P, Ryder REJ. Is digital image compression acceptable within diabetic reitnopathy screening. Diabet Med 2003; 20: 766-771.

3 Sung MM, Kim HJ, Yoo SK, Choi BW, Nam JE, Kim HS et al. Clinical evaluation of compression ratios using JPEG2000 on computed radiography chest images. J Digit Imaging 2002; 15: 78-83.

4 Fidler A, Likar B, Pernus F, Skaleric U. Comparative evaluation of JPEG and JPEG2000 compression in quantitative digital subtraction radiography. Dentomaxillofac Radiol 2002; 31: 379-384.

5 Landis JR, Koch GG. The measurement of observer agreement for categorical data. Biometrics 1977; 33: 159-174.

6 Christopoulos C, Skodras A, Ebrahimi T. The JPEG2000 still image coding: an overview. IEEE Trans Consumer Electron 2000; 46: 1103-1127.

7 Marcellin MW, Gormish MJ, Bilgin A, Boliek MP. An overview of JPEG-2000, in Proceedings of 2000 Data Compression Conference, pp 523-544 March 2000.

8 Eikelboom RH, Yogesan K, Barry CJ, Constable IJ, TayKearney ML, Jitskaia L et al. Methods and limits of digital image compression of retinal images for telemedecine. Invest Ophthalmol Vis Sci 2000; 41: 1916-1924.

9 Newsom RS, Clover A, Costen MT, Sadler J, Newton J, Luff $\mathrm{AJ}$ et al. Effect of digital image compression on screening for diabetic retinopathy. Br J Ophthalmol 2001; 85: 799-802.

10 Stellingwerf C, Hardus PL, Hooymans JM. Assessing diabetic retinopathy using two-field digital photography and the influence of JPEG-compression. Doc Ophthalmol 2004; 108: 203-209.

11 Baker CF, Rudnisky CJ, Tennant MT, Sanghera P, Hinz BJ, De Leon AR et al. JPEG compression of stereoscopic digital images for the diagnosis of diabetic retinopathy via teleophthalmology. Can J Ophthalmol 2004; 39: 746-754.

12 Persons KR, Palisson P, Manduc A, Erickson BJ, Savcenko V. An analytical look at the effects of compression on medical images. J Digit Imag 1997; 10: 60-66.

13 Klein R, Meuer SM, Moss SE, Klein BE. Retinal microaneurysm counts and 10-year progression of diabetic retinopathy. Arch Ophthalmol 1995; 113: 1386-1391.

14 George LD, Halliwell M, Hill R, Aldington SJ, Lusty J, Dunstan $\mathrm{F}$ et al. A comparison of digital retinal images and $35 \mathrm{~mm}$ colour transparencies in detecting and grading diabetic retinopathy. Diabet Med 1998; 15: 250-253.

15 Kerr D, Cavan DA, Jennings B, Dunnington C, Gold D, Crick $M$. Beyond retinal screening: digital imaging in the assessment and follow-up of patients with diabetic retinopathy. Diabet Med 1998; 15: 878-882.

16 Lin DY, Blumenkrantz MS, Brothers R. The role of digital fundus photography in diabetic retinopathy screening. Digital Diabetic Screening Group (DDSG). Diabetes Technol Ther 1999; 1: 4477-4487.

17 Henricsson M, Karlsson C, Ekholm L, Kaikkonen P, Sellman A, Steffert E et al. Colour slides or digital photography in diabetes screening - a comparison. Acta Ophthalmol Scand 2000; 78: 164-168.

18 Massin P, Erginay A, Ben Mehidi A, Vicaut E, Quentel G, Victor $\mathrm{Z}$ et al. Evaluation of a new non-mydriatic digital camera for detection of diabetic retinopathy. Diabet Med 2003; 20: 635-641.

19 Lim JI, LaBree L, Nichols T, Cardenas I. A comparison of digital nonmydriatic fundus imaging with standard 35-millimeter slides for diabetic retinopathy. Ophthalmology 2000; 107: 866-870. 